Communicated by William W. Lytton

\title{
Neural Network Modeling of Memory Deterioration in
} Alzheimer's Disease

\section{Horn}

School of Physics and Astronomy, Raymond and Beverly Sackler Faculty of

Exact Sciences, Tel Aviv University, Tel Aviv 69978, Israel

\section{E. Ruppin}

Department of Computer Science, Raymond and Beverly Sackler Faculty of

Exact Sciences, Tel Aviv University, Tel Aviv 69978, Israel

\section{Usher}

CNS program, Division of Biology 216-76, Caltech, Pasadena, CA 91125 USA

\section{Herrmann}

Sektion Informatik, Universität Leipzig, PSF 920, D-0-7010 Leipzig, Germany

The clinical course of Alzheimer's disease (AD) is generally characterized by progressive gradual deterioration, although large clinical variability exists. Motivated by the recent quantitative reports of synaptic changes in AD, we use a neural network model to investigate how the interplay between synaptic deletion and compensation determines the pattern of memory deterioration, a clinical hallmark of AD. Within the model we show that the deterioration of memory retrieval due to synaptic deletion can be much delayed by multiplying all the remaining synaptic weights by a common factor, which keeps the average input to each neuron at the same level. This parallels the experimental observation that the total synaptic area per unit volume (TSA) is initially preserved when synaptic deletion occurs. By using different dependencies of the compensatory factor on the amount of synaptic deletion one can define various compensation strategies, which can account for the observed variation in the severity and progression rate of AD.

\section{Introduction}

Alzheimer's disease (AD) is the major degenerative disease of the brain, responsible for a progressive deterioration of the patient's cognitive and motor function, with a grave prognosis (Adams and Victor 1989). Its clinical course is usually characterized by gradual decay, although both slow and rapidly progressive forms have been reported, exhibiting a large

Neural Computation 5, 736-749 (1993) (C) 1993 Massachusetts Institute of Technology 
variation in the rate of $\mathrm{AD}$ progression (Drachman et al. 1990). While remarkable progress has been gained in the investigation of neurochemical processes accompanying $\mathrm{AD}$, their role in neural degeneration, the main pathological feature of AD, is yet unclear (Selkoe 1987; Kosik 1991). This work is motivated by recent investigations studying in detail the neurodegenerative changes accompanying $\mathrm{AD}$, on a neuroanatomical level. Following the paradigm that cognitive processes can be accounted for on the neural level, we examine the effect of these neurodegenerative changes within the context of a neural network model. This allows us to obtain a schematic understanding of the clinical course of AD.

Neuroanatomical investigations in AD patients demonstrate a considerable decrease in the synapse to neuron ratio, due to synaptic deletion (Davies et al. 1987; Bertoni-Freddari et al. 1990). Synaptic compensation, manifested by an increase of the synaptic size, was found to take place concomitantly, reflecting a functional compensatory increase of synaptic efficacy at the initial stages of the disease (Bertoni-Freddari et al. 1988, 1990; DeKosky and Scheff 1990). The combined outcome of these counteracting synaptic degenerative and compensatory processes can be evaluated by measuring the total synaptic area per unit volume (TSA), which was shown to correlate with the cognitive function of $\mathrm{AD}$ patients (DeKosky and Scheff 1990).

Our model, presented in Section 2, serves as a framework for examining the interplay of synaptic deletion and compensation. This attractor neural network (ANN) is not supposed to represent any specific neuronal tissue, yet we believe that our results are relevant to a large class of neural systems. Deletion is carried out stochastically by removing the fraction $d$ of all synaptic weights. Compensation is modeled by multiplying all remaining synaptic weights by a common factor $c$. The TSA value is proportional to $c(1-d)$. Varying $c$ as a function of $d$ specifies a compensation strategy. We assume that the network's failure rate, measured by the fraction of memories erroneously retrieved, represents the degree of "cognitive deficit" in clinical observations. Reviewing the pertaining pathological and clinical data, we show in Section 3 how our model can account for the variability observed in the clinical course of AD. Our results are further discussed in Section 4.

\section{The Model}

Concentrating on memory degradation, a clinical hallmark of AD (Adams and Victor 1989), we use as our theoretical framework a neural network model of associative memory. Our model is based on the biologically motivated variant of Hopfield's model (1982), proposed by Tsodyks and Feigelman (1988). In an ANN, the stored memories are attractors of the network's dynamics, such that when memory retrieval is modeled then, starting from an initial condition sufficiently similar to one of the memory 
patterns, the network flows to a stable state identical with that memory. The appeal of attractors, as corresponding to our intuitive notion of the persistence of cognitive concepts along some temporal span, has been fortified by numerous studies testifying to the applicability of ANNs as models of the human memory [for a review see Amit (1989)], and is also supported by biological findings of delayed, poststimulus, sustained activity (Fuster and Jervey 1982; Miyashita and Chang 1988).

All $N$ neurons in the network have a uniform positive threshold $T$. Each neuron is described by a binary variable $S=\{1,0\}$ denoting an active (firing) or passive (quiescent) state, respectively. $M=\alpha N$ distributed memory patterns $\xi^{\mu}$ are stored in the network. The elements of each memory pattern are chosen to be $1(0)$ with probability $p(1-p)$ respectively, with $p \ll 1$. The weights of the synaptic connections are

$$
W_{i j}=\frac{1}{N} \sum_{\mu=1}^{M}\left(\xi_{i}^{\mu}-p\right)\left(\xi_{j}^{\mu}-p\right)
$$

The updating rule for neuron $i$ at time $t$ is given by

$$
S_{i}(t)=\Theta\left[\sum_{j} W_{i j} S_{j}(t-1)-T\right]
$$

where $\Theta$ is the step function. The performance of the network is measured by the activities of the memories, as defined by the overlaps $m^{\mu}$,

$$
m^{\mu}(t)=\frac{1}{p(1-p) N} \sum_{i=1}^{N}\left(\xi_{i}^{\mu}-p\right) S_{i}(t)
$$

As shown in the Appendix, there exists an optimal value of the threshold $T=p(1-p)(1-2 p) / 2$, which ensures the best performance of the network.

Starting with such a memory model we introduce synaptic deletion by randomly deleting some of the incoming synapses of every neuron, leaving each neuron with $l=(1-d) N$ input connections, where $d<1$ is the deletion factor. Synaptic compensation is modeled by multiplying the weights of the remaining synaptic connections by a uniform compensation factor $c>1$. This changes the dynamics of the system to

$$
S_{i}(t)=\Theta\left[\sum_{j \in D_{i}} c W_{i j} S_{j}(t-1)-T\right]
$$

where $D_{i}$ denotes a random set of indices corresponding to neurons to which the $i$ th neuron is connected, and $\left|D_{i}\right| / N=1-d \leq 1$. T remains the same value as before.

In the network's "premorbid" state, the memories have maximal stability, achieved by choosing the optimal threshold $T$ that maximizes the increase of the overlap (say $m^{1}$ ), as shown in the Appendix. When the network is initialized with an input pattern that is a corrupted version 


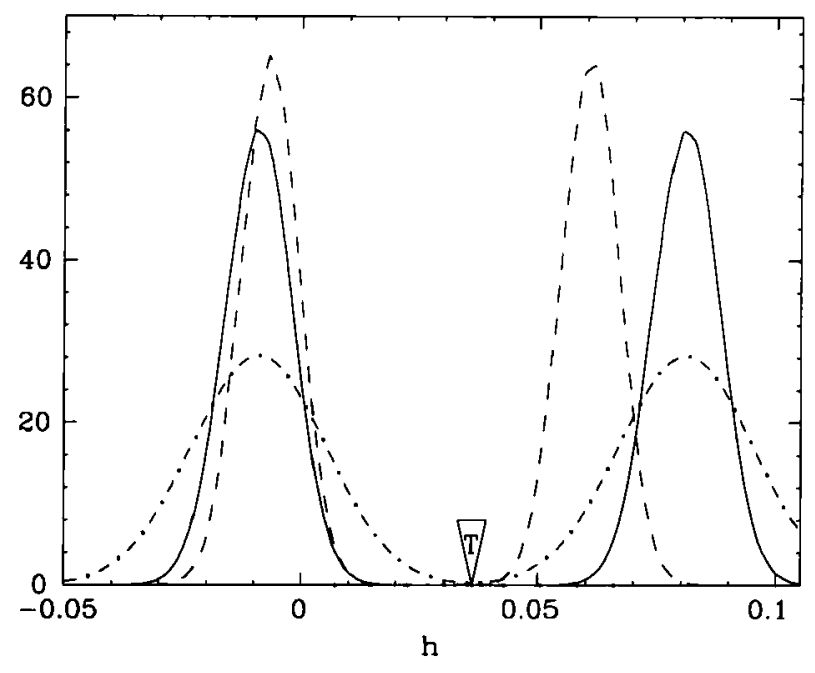

Figure 1: The distribution of the postsynaptic potential ( $p=0.1, \alpha=0.05$ ). Solid curve: Initial state: two gaussian distributions peaked at $-p^{2}(1-p)$ and $p(1-p)^{2}$. The optimal threshold $T=p(1-p)\left(\frac{1}{2}-p\right)$ lies in the middle between the two gaussian mean values. Dashed curve: After deletion $(d=0.25)$, the new peaks of the postsynaptic potential are no longer equidistant from the threshold $T$. Dot-dashed curve: The OPC strategy restores the initial mean values of the postsynaptic potential $(d=0.75)$.

of one of the stored memory patterns (e.g., $\xi^{1}$ ), it will flow dynamically into the attractor given by this memory.

To obtain an intuitive notion of the network's behavior when synaptic deletion and compensation are incorporated consider Figure 1. The neurons that stand for firing neurons in the stored memory, and the neurons that stand for quiescent neurons in the stored memory, have distinct postsynaptic potential distributions (the solid curves in Fig. 1). When synaptic deletion takes place, the mean values of the neurons' postsynaptic potential change, and the threshold is no longer optimal (see dashed curves in Fig. 1). Multiplying the weights of the remaining synaptic connections by an optimal performance compensation factor (OPC) $c=1 /(1-d)$, restores the original mean values of postsynaptic potential and the optimality of the threshold (dot-dashed curves in Fig. 1). The accompanying increase in the variance of the postsynaptic potential, which is $\sqrt{1 /(1-d)}$ times larger than the original one, leads, however, to performance deterioration. This is further elucidated in the Appendix. 


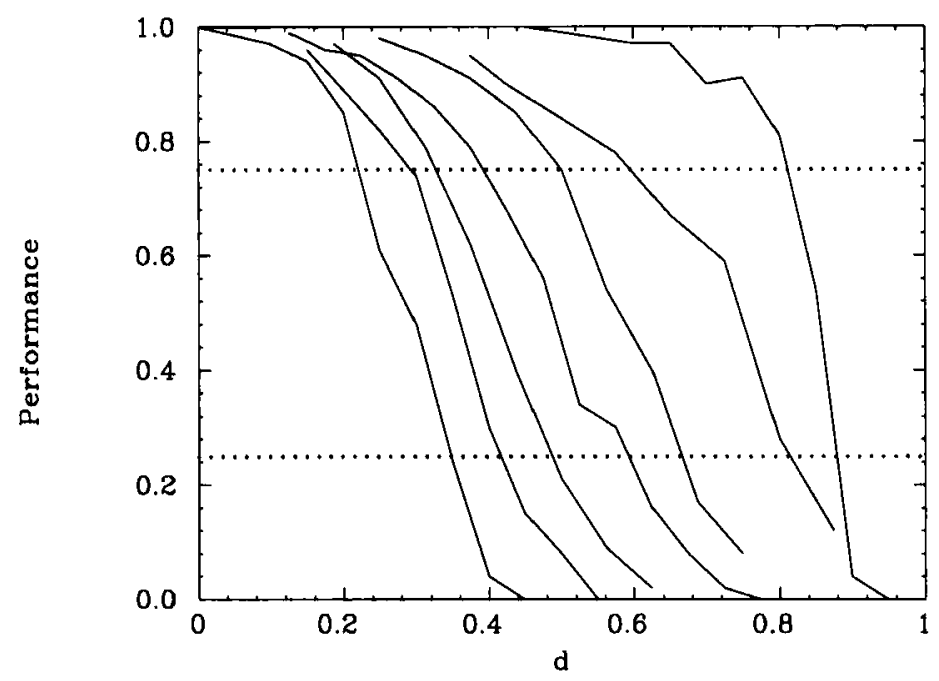

Figure 2: Performance of a network with fixed $k$ compensation. Starting from an initial state that is a corrupted version $\left[\mathrm{m}^{1}(0)=0.8\right]$ of a stored memory pattern, we define performance as the percentage of cases in which the network converged to the correct memory. The simulation parameters are $N=800$ neurons, $\alpha=0.05$, and $p=0.1$. The curves represent (from left to right) the performance of fixed strategies with increasing $k$ values, for $k=0,0.25,0.375,0.5,0.625,0.75,1$. The horizontal dotted lines represent performance levels of 25 and $75 \%$.

We can interpolate between the case of deletion without compensation and the OPC within a class of compensatory strategies, defined by

$$
\hat{W}_{i j}=c W_{i j}, \quad c=1+\left(\frac{1}{1-d}-1\right) k=1+\frac{d k}{1-d}
$$

with the parameter $0 \leq k \leq 1$. All the fixed $k$ strategies, examined via simulations measuring the performance of the network at various deletion and compensation levels, display a similar sharp transition from the memory-retrieval phase to a nonretrieval phase, as shown in Figure 2. Varying the compensation magnitude $k$ merely shifts the location of the transition region. This sharp transition is similar to that reported previously in cases of deletion without compensation in other models (Canning and Gardner 1988; Koscielny-Bunde 1990). 


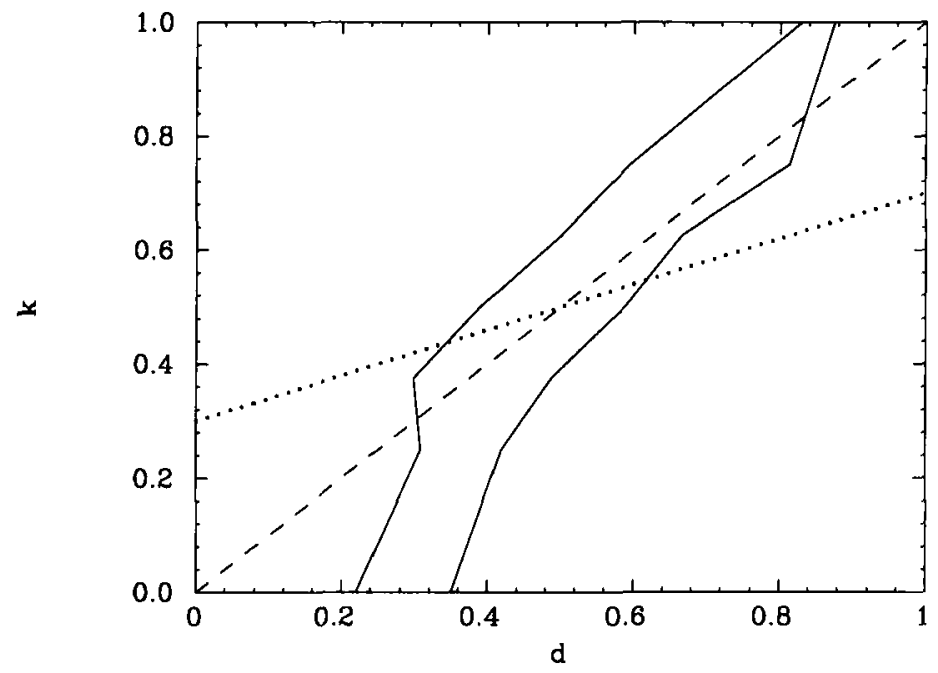

Figure 3: The critical transition range in the $(k, d)$ plane. The solid curves represent performance levels of 75 and $25 \%$, derived from Figure 2. The straight lines describe the variations employed in two variable compensations presented in Figure 4.

Figure 3 describes the transition region as a map in the $(k, d)$ plane. The performance levels read off Figure 2 delineate the domain over which deterioration occurs. Staying close to the upper boundary of this domain defines a compensation strategy that enables the system to maintain its performance, with a much smaller amount of synaptic strengthening than that required by the OPC strategy. In the face of restricted compensatory resources, such an optimal resource compensation strategy (ORC) could be of vital importance. The essence of such ORC strategy is that $k$ is varied as synaptic deletion progresses, in order to retain maximal performance with minimal resource allocation. In Figure 4, we present the performance of two variable $k$ compensation strategies, which we propose to view as expressions of (albeit unsuccessful) attempts of maintaining an ORC. These examples, indicated in Figure 3, include a "gradually decreasing" strategy defined by the variation $k=0.3+0.4 d$, and the "plateau" strategy defined by the variation $k=d$. The analogs of these strategies can be found in clinical observations, as shown in the next section where we review the biological and clinical evidence relevant to our model. 


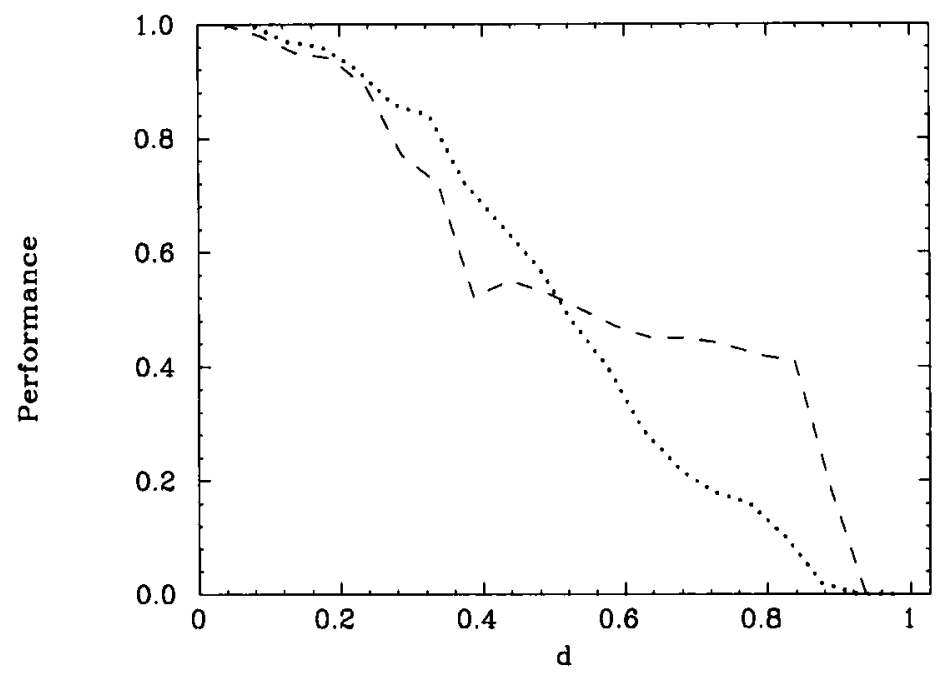

Figure 4: Performance of a network with gradually decreasing (dotted curve) and plateau (dashed curve) compensation strategies.

\section{Clinical Motivation and Implications}

As mentioned in the introduction, while synaptic degeneration occurs, the TSA stays constant in some cortical layers at the initial stages of AD. Qualitatively similar synaptic changes have been observed during normal physiological aging, but with significantly lower deletion (BertoniFreddari et al. 1988, 1990). Hence a plausible general scenario seems to involve some initial period of OPC. As AD progresses, synaptic compensation no longer succeeds in maintaining the TSA (Bertoni-Freddari et al. 1990; DeKosky and Scheff 1990). In advanced AD cases, severe compensatory dysfunction has been observed (Buell and Coleman 1979; Flood and Coleman 1986; DeKosky and Scheff 1990).

Young AD patients are likely to have high compensation capacities, and therefore can maintain an OPC strategy ( $k=1$, in Fig. 2) throughout the course of their disease. This will then lead to a rapid deterioration when the reserve of synaptic connections has been depleted. Indeed, young AD patients have been reported to have a rapid clinical progression (Heston et al. 1981; Heyman et al. 1983), accompanied by severe neuronal and synaptic loss (Hansen et al. 1988). A similar clinical pattern of rapid memory decline, already manifested with less severe neuroanatomical pathology, was found in very old patients (Huff et al. 1987). 
We propose that in these old patients, the rapid clinical decline results from the lack of compensatory capacities ( $k=0$, in Fig. 2), possibly of the kind observed by Buell and Coleman (1979) and Flood and Coleman (1986).

Rapid cognitive decline characterizes a minority of AD patients. Most patients show a continuous gradual pattern of cognitive decline (Adams and Victor 1989; Katzman 1986; Katzman et al. 1988), taking place along a broad span of synaptic deletion (DeKosky and Scheff 1990). As shown in Figure 2, this performance decline cannot be accounted for by any network employing fixed $k$ compensation. Variable compensation, such as that defined by the gradually decreasing strategy, is needed to explain the memory decline observed in the majority of $A D$ patients, as shown in Figure 4. The clinical state of some AD patients remains stable at mild to moderate levels for several years before finally rapidly decreasing (Cummings and Benson 1983; Katzman 1985; Botwinick et al. 1986). This can be accounted for by a "plateau" strategy whose performance, shown in Figure 4, stays at an approximately constant level over a large domain of deletion.

Synaptic deletion and compensatory mechanisms play a major role also in the pathogenesis of Parkinson disease (Zigmond et al. 1990; Calne and Zigmond 1991). The significant incidence of $A D$ patients having accompanying extrapyramidal parkinsonian signs (Mayeux et al. 1985; Stern et al. 1990) naturally raises the possibility that such patients may have a decreased synaptic compensatory potential in general (Horn and Ruppin 1992). The cognitive deterioration of these AD patients is faster than that of AD patients without extrapyramidal signs. This fits well with our proposal that severely deteriorated synaptic compensation capacity leads to an accelerated rate of cognitive decline in $A D$ patients. This issue is still inconclusive because the PD-AD combination may be a specific syndrome on its own.

\section{Discussion}

In accordance with the findings that neuronal loss in $A D$ is less than $10 \%$ even at advanced stages (Katzman 1986), and that the synapse to neuron ratio is significantly decreased (Davies et al. 1987; Bertoni-Freddari et al. 1990), we have concentrated on studying the role of the synaptic changes. Simulations we have performed incorporating neuronal loss have shown similar results to those presented above. We conclude therefore that the important factors are indeed the number of synapses retained and the compensation strategy employed, whose interplay may lead to various patterns of performance decline.

As any current neural model of human cognitive phenomena, our model necessarily involves many simplifications. The TF formal neurons are obviously a very gross simplification of biological neurons. As in 
most Hopfield-like ANNs, the network has no spatially specified architecture. For clarity of exposition of our main ideas, we have assumed that all compensation strategies are applied uniformly to all retained synapses. Our analysis also holds for nonuniform compensation, that is, when each remaining synaptic weight is multiplied by a random variable with mean value $c$ and variance $\sigma^{2}$, since the same averages of the postsynaptic potentials are obtained (see Fig. 1 and the Appendix). Obviously, if the variance is too large, then no compensation strategy can be conceived of any more. Motivated by the biological evidence testifying to the sparsity of neural firing (Abeles et al. 1990), we have assumed a relatively small fraction $p$ of firing neurons. Simulations performed with higher $p$ values (e.g., 0.2 ) indicate that the results remain qualitatively the same. However, it should be noted that as $p$ is increased the approximation of the network's overlap dynamics presented in the Appendix becomes less and less accurate.

The variable compensation strategies that we have discussed rely on the fact that there is some span in the $(k, d)$ plane over which deterioration takes place, as shown in Figure 3. As $N$ is increased, the width of the domain over which deterioration occurs keeps getting narrower, thus limiting the possibilities of maneuvering between deletion and compensation. Hence, one may claim that our conclusions, which are based on simulations of small scale networks, do not hold for the brain.

One possible answer to this problem is that there may exist important modules in the brain whose function depends on the correct performance of just some thousands of neurons (Eccles 1981). For large cortical modules, this objection may be resolved by considering the effect of noise present in the brain. To account for the latter, any realistic paradigm of memory recall should entail the recognition of a spectrum of noisy inputs presented to the network. Figure 5 displays the performance of the network in the $(k, d)$ plane obtained via simulations with two distinct initial overlap values $\left[m^{1}(0)=0.8\right.$ and $\left.m^{1}(0)=0.95\right]$, together with the theoretical results for the infinite $N$ limit. These results show that even in this limit, the corresponding performance curve always retains a finite width as long as the network processes input patterns with a broad range of initial overlaps. Consequently, the realization of variable compensatory strategies may indeed be feasible in the brain.

The decline in the network's performance resulting from synaptic deletion is coupled with a decrease in the network's overall activity. This observation gives rise to the possibility that although being defined "globally" as "strategies," synaptic compensation may take place via local feedback mechanisms. Accordingly, the decreased firing rate of a neuron being gradually deprived of its synaptic inputs may enhance the activity of cellular processes strengthening its remaining synapses. This scenario seems to lead to fixed OPC compensation in a rather straightforward manner, but as synaptic deletion may be nonhomogeneous the effects of the resulting spatially nonuniform compensatory changes should be fur- 


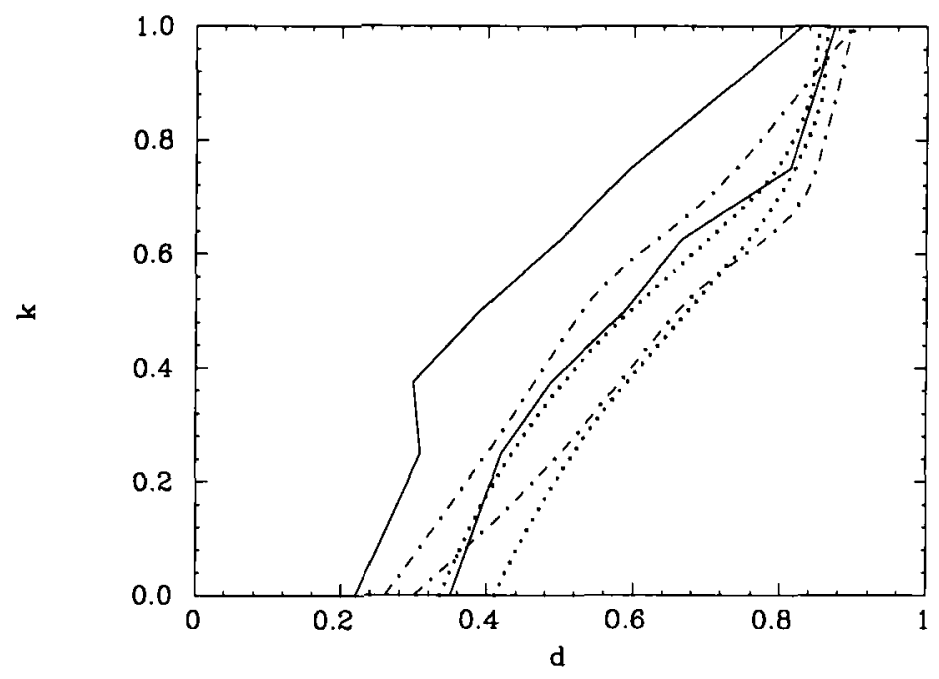

Figure 5: The critical transition range in the $(k, d)$ plane. The solid curves represent performance levels of 75 and $25 \%$, with initial overlap $m(0)=0.8$ (identical to Fig. 2). The dash-dotted curves represent performance levels of 75 and $25 \%$, for initial overlap $m(0)=0.95$. The dotted curves represent the theoretical results that follow from the analysis presented in the Appendix, delineating the estimates of when the corresponding basins of attraction cease to exist in the infinite $N$ limit. These curves lie close to the $25 \%$ lines of the simulations.

ther investigated. The nonvanishing width of the $(k, d)$ plane transition range shown above is essential for the feasibility of an ORC strategy, so that local mechanisms can "trace" the decreasing performance and "counteract" it before the performance collapses entirely.

Finally let us comment on possible examinations and applications of our model. An ideal experiment would involve a series of consecutive measurements of synaptic strength and cognitive abilities. In light of obvious difficulties concerning such tests, we may have to resort to comparing biopsies and autopsies, as in DeKosky and Scheff (1990), preferably on the same patients. Our model demonstrates the importance of maintaining the TSA for the preservation of memory capacity and, therefore, mental ability of $\mathrm{AD}$ patients. This may suggest that future therapeutic efforts in AD should include an attempt to mobilize compensatory mechanisms facilitating the regeneration of synaptic connectivity. 


\section{Appendix}

Qualitative features of our model can be derived from a simple analysis of the first iteration step. Starting with a state that is close to $\xi^{1}$ with overlap $m^{1}(0)$ we wish to find whether the network flows into the correct memory. Using the dynamics defined in the text we find for $t=1$

$$
\begin{aligned}
S_{i}(1) & =\Theta\left\{\frac{1}{N} c\left[\sum_{j \in D_{i}}\left(\xi_{i}^{1}-p\right)\left(\xi_{j}^{1}-p\right) S_{j}+\sum_{\mu>1} \sum_{j \in D_{i}}\left(\xi_{i}^{\mu}-p\right)\left(\xi_{j}^{\mu}-p\right) S_{j}\right]-T\right\} \\
& =\Theta\left[c(1-d)\left(\xi_{i}^{1}-p\right) p(1-p) m^{1}(0)+\mathbf{N}-T\right]
\end{aligned}
$$

where we have separated the signal from the noise term. The latter has zero average, $\langle\mathbf{N}\rangle=0$, and variance of $\left\langle\mathbf{N}^{2}\right\rangle=c^{2}(1-d) p^{2}(1-p)^{2} \alpha s(0)$, where $s(0)=P\left[S_{i}(0)=1\right]=1-p-m^{1}(0)+2 p m^{1}(0)$. In view of the gaussian noise term we write the probability in terms of an error-function,

$$
P\left(S_{i}=1\right)=\operatorname{erf}\left[\frac{c(1-d)\left(\xi_{i}^{1}-p\right) p(1-p) m^{1}(0)-T}{\sqrt{c^{2}(1-d) \alpha s(0) p^{2}(1-p)^{2}}}\right]
$$

This results in the following expression for the first iteration:

$$
\begin{aligned}
m^{1}(1)= & \frac{1}{p(1-p)}\left\{(1-p) P\left(\xi_{i}^{1}=1\right) P\left[S_{i}(1)=1 \mid \xi_{i}^{1}=1\right]\right. \\
& \left.-p P\left(\xi_{i}^{1}=0\right) P\left[S_{i}(1)=1 \mid \xi_{i}^{1}=0\right]\right\} \\
= & P\left[S_{i}(1)=1 \mid \xi_{i}^{1}=1\right]-P\left[S_{i}(1)=1 \mid \xi_{i}^{1}=0\right] \\
= & \operatorname{erf}\left[\frac{(1-p) m^{1}(0) p(1-p) c(1-d)-T}{\sqrt{\alpha c^{2}(1-d) s(0) p^{2}(1-p)^{2}}}\right] \\
& -\operatorname{erf}\left[\frac{(-p) m^{1}(0) p(1-p) c(1-d)-T}{\sqrt{\alpha c^{2}(1-d) s(0) p^{2}(1-p)^{2}}}\right]
\end{aligned}
$$

In the limit $m^{1}(0) \rightarrow 1$ one finds the maximal value of equation A.3 to be obtained for the following choice of the optimal threshold:

$$
T^{*}=c(1-d) p(1-p)(1-2 p) / 2
$$

For $c=1$ and $d=0$ this coincides with the choice we have made. Moreover, as long as

$$
c=\frac{1}{1-d}
$$

which was defined as the OPC strategy, $T$ remains optimal. This fact was expressed graphically in Figure 1. The two gaussian distributions in this figure correspond to the two terms in equation A.3. 
In the simulations we have looked for the cases in which the system converged onto the correct fixed points. This involves iterating the equations of motion, which is in general different from iterating expressions like equation A.3 because of possible correlations between the different time steps. Nonetheless we may think of the iteration of equation A.3 (replacing the labeling 0 and 1 by $n-1$ and $n$ ) as a rough estimate for the strongly diluted infinite system (Evans 1989). Starting out with different values for $m^{1}(0)$ we find the dotted curves in Figure 5, which show the borderlines between convergence and nonconvergence to the correct memory.

An alternative to this derivation is to use the replica symmetry assumption. We have carried out such an analysis (Herrmann et al. 1992). The results are similar, though not identical, to the ones reported above.

\section{References}

Abeles, M., Vaadia, E., and Bergman, H. 1990. Firing patterns of single units in the prefrontal cortex and neural network models. Network 1, 13-25.

Adams, R. D., and Victor, M. 1989. Principles of Neurology. McGraw-Hill, New York.

Amit, D. J. 1989. Modeling Brain Function: The World of Attractor Neural Networks. Cambridge University Press, Cambridge.

Bertoni-Freddari, C., Meier-Ruge, W., and Ulrich, J. 1988. Quantitative morphology of synaptic plasticity in the aging brain. Scanning Microsc. 2, 1027-1034.

Bertoni-Freddari, C., Fattoretti, P., Casoli, T., Meier-Ruge, W., and Ulrich, J. 1990. Morphological adaptive response of the synaptic junctional zones in the human dentate gyrus during aging and Alzheimer's disease. Brain Res. 517, 69-75.

Botwinick, J., Storandt, M., and Berg, L. 1986. A longitudinal behavioral study of senile dementia of the Alzheimer type. Arch. Neurol. 43, 1124-1127.

Buell, S. J., and Coleman, P. D. 1979. Dendritic growth in the aged human brain and failure of growth in senile dementia. Science 206, 854-856.

Calne, D. B., and Zigmond, M. J. 1991. Compensatory mechanisms in degenerative neurologic diseases. Arch. Neurol. 48, 361-363.

Canning, A., and Gardner, E. 1988. Partially connected models of neural networks. J. Phys. A: Math. Gen. 214, 3275-3284.

Cummings, J. L., and Benson, D. F. 1983. Dementia: A Clinical Approach. Butterworths, London.

Davies, C. A., Mann, D. M. A., Sumpter, P. Q., and Yates, P. O. 1987. A quantitative morphometric analysis of the neuronal and synaptic content of frontal and temporal cortex in patient with Alzheimer's disease. J. Neurol. Sci. 78, $151-164$.

DeKosky, S. T., and Scheff, S. W. 1990. Synapse loss in frontal cortex biopsies in Alzheimer's disease: Correlation with cognitive severity. Ann. Neurol. 27(5), 457-464. 
Drachman, D. A., O'Donnell, B. F., Lew, R. A., and Swearer, J. M. 1990. The prognosis in Alzheimer's disease. Arch. Neurol. 47, 851-856.

Eccles, J. C. 1981. The modular operation of the cerebral neocortex considered as the material basis of mental events. Neuroscience 6, 1839-1855.

Evans, M. R. 1989. Random dilution in a neural network for biased patterns. J. Phys. A: Math. Gen. 22, 2103-2118.

Flood, D. G., and Coleman, P. D. 1986. Failed compensatory dendritic growth as a pathophysiological process in Alzheimer's disease. Can. J. Neurol. Sci. $13,475-479$.

Fuster, J. M., and Jervey, J. P. 1982. Neuronal firing in the inferotemporal cortex of the monkey in a visual memory task. J. Neurosci. 2(3), 361-375.

Hansen, L. A., DeTeresa, R., Davies, P., and Terry, R. D. 1988. Neocortical morphometry, lesion counts, and choline acetyltransferase levels in the age spectrum of Alzheimer's disease. Neurology 38, 48-54.

Herrmann, M., Horn, D., Ruppin, E., and Usher, M. 1992. Variability in the pathogenesis of Alzheimer's disease: analytical results. To appear in the Proc. ICANN*92, September, Brighton, UK (in press).

Heston, L. L., Mastri, A. R., Anderson, V. E., and White, J. 1981. Dementia of the Alzheimer type: clinical genetics, natural history, and associated conditions. Arch. Gen. Psychiat. 38, 1085-1090.

Heyman, A., Wilkinson, W. E., Hurwitz, P. J., Schmechel, D., Sigmon, A. H., Weinberg, T., Helms, M. J., and Swift, M. 1983. Alzheimer's disease: Genetic aspects and associated clinical disorders. Ann. Neurol. 14(5), 507-515.

Hopfield, J. J. 1982. Neural networks and physical systems with emergent collective abilities. Proc. Natl. Acad. Sci. U.S.A. 79, 2554-2558.

Horn, D., and Ruppin, E. 1992. Extra-pyramidal symptoms in Alzheimer's disease: a hypothesis. Med. Hypotheses 39(4), 316-318.

Huff, F. J., Growden, J. H., Gorkin, S., and Rosen, T. J. 1987. Age of onset and rate of progression of Alzheimer's disease. J. Am. Geriatr. Soc. 35, 27-30.

Jansen, K. L. R., Faull, R. L. M., Dragunow, M., and Synek, B. L. 1990. Alzheimer's disease: Changes in hippocampal $N$-methyl-D-aspartate, quisqualate, neurotensin, adenosine, benzodiazepine, serotonin and opoid receptors-an autoradiographic study. Neuroscience 39(3), 613-627.

Katzman, R. 1985. Clinical presentation of the course of Alzheimer's disease: The atypical patient. Interdiscipl. Topics. Gerontol. 20, 12-18.

Katzman, R. 1986. Alzheimer's disease. N. Engl. J. Med. 314(15), 964-973.

Katzman, R., et al. 1988. Comparison of rate of annual change of mental status score in four independent studies of patients with Alzheimer's disease. Ann. Neurol. 24(3), 384-389.

Koscielny-Bunde, E. 1990. Effect of damage in neural networks. J. Statist. Phys. 58, 1257-1266.

Kosik, K. S. 1991. Alzheimer's plaques and tangles: Advances in both fronts. TINS 14, 218-219.

Mayeux, R., Stern, Y., and Spanton, S. 1985. Heterogeneity in dementia of the Alzheimer type: Evidence of subgroups. Neurology 35, 453-461.

Miyashita, Y., and Chang, H. S. 1988. Neuronal correlate of pictorial short-term memory in the primate temporal cortex. Nature (London) 331, 68. 
Selkoe, D. J. 1987. Deciphering Alzheimer's disease: The pace quickens. TINS 10, $181-184$.

Stern, Y., Hesdorffer, D., Sano, M., and Mayeux, R. 1990. Measurement and prediction of functional capacity in Alzheimer's disease: Neurology 40, 8-14.

Tsodyks, M. V., and Feigelman, M. V. 1988. The enhanced storage capacity in neural networks with low activity level. Europhys. Lett. 6, 101-105.

Zigmond, M. J., Abercrombie, E. D., Berger, T. W., Grace, A. A., and Stricker, E. M. 1990. Compensations after lesions of central dopaminergic neurons: Some clinical and basic implications. TINS 13, 290.

Received 1 July 1992; accepted 14 January 1993. 